\title{
Lexicology of remuneration in French. Between legal and familiar French
}

\section{[Le lexique des rémunérations en français. Entre la langue juridique et la langue familière]}

\author{
Pierre Brouland - Janka Priesolova
}

DOI: 10.18355/XL.2016.09.02.57-72

Résumé

L'article est une contribution à l'étude de la terminologie du droit du travail en français en confrontation avec le tchèque. Il propose une analyse linguistique des rémunérations en français, sujet qui n'a pas encore fait l'objet d'une étude spécialisée. Il se donne pour but de donner une description exhaustive de la terminologie des différents types de rémunération en français (du point de vue juridique et linguistique, au niveau de la forme, du contenu, des concepts) dans leur évolution historique et du point de vue contrastif en comparaison avec le tchèque. À la différence du tchèque le lexique des rémunérations apparait en français d'une extrême complexité car il relève à la fois de la langue juridique qui entend définir clairement chaque type de revenus, mais aussi de la langue familière, chaque corps de métier ayant développé des expressions correspondant à sa propre situation. Nous attirons l'attention sur la diversité de la terminologie française ainsi que sur la polysémie et l'ambiguïté des termes clé dans les deux langues en proposant leurs équivalents (partiels ou fonctionnels) notamment dans le cas où leurs dénotations ne se recouvrent pas ou quand ils n'ont pas d'équivalent formel spécialisé dans la langue cible (descriptions, paraphrases explicatives).

Mots clés : lexique juridique français, rémunérations, salaire, professions, équivalence fonctionnelle

\section{Introduction}

Il est fréquent de considérer le lexique du droit comme le reflet d'un système juridique. Pour cette raison, ce vocabulaireapporte des indications précieuses sur les valeurs d'une société donnée (ou du moins sur l'idéologie de ses élites) ainsi que sur leurs évolutions. Ce champ lexical comprend des termes juridiques au sens propres du terme, ainsi que leurs collocations, mais aussi des mots de double appartenance avec des expressions issues de la langue courante dans des combinaisons syntagmatiques spécialisées. La rémunération est un des domaines du droit du travail qui concerne directement toutes les personnes intégrées au monde du travail. Il s'agit là d'une question primordiale étroitement liée à la vie de tous les jours. Pour cette raison, la langue juridique ayant trait à cette thématique a été enrichi par des moyens d'expression populaires et même argotiques. Le lexique des rémunérations apparait ainsi en français d'une extrême complexité car il relève à la fois de la langue juridique qui entend définir clairement chaque type de revenus, mais aussi de la langue familière, chaque corps de métier ayant développé des expressions correspondant à sa propre situation.

Dans notre article, nous nous donnons pour but de recenser, de façon aussi exhaustive que possible, la terminologie des différents types de rémunération en français (non seulement du point de vue juridique et linguistique, mais aussi au niveau

XLinguae Journal, Volume 9 Issue 2, April 2016, ISSN 1337-8384 
de la forme et du contenu) dans leur évolution historique ainsi que du point de vue contrastif en comparaison avec le tchèque. In fine, nous proposerons quelques équivalents tchèques des termes français clés. Cet article analysant la problématique de la rémunération en français qui n'a pas encore été l'objet d'une étude spécialisée, fait partie d'un projet de recherche (IGA no 52/2014) réalisé à l'Université d'Économie de Prague consacré à la « Terminologie française du droit du travail et de la sécurité sociale $»$.

\section{I.Rémunération, rétribution ou salaire?}

Les deux mots les plus utilisés en français pour désigner la "rétribution » d'un travail sont salaire et rémunération. Jusqu'à la fin du XVIII ${ }^{\mathrm{e}}$ siècle, les deux expressions ont pu être considérées comme des synonymesquasi parfaits. Avec la révolution industrielle, le mot salaire a fini par prendre un sens plus étroit, et ne renvoie plus aujourd'hui qu'à la somme due par l'employeur au salarié en vertu d'un contrat de travail.Néanmoins, nous pourrons constater que certaines ambiguïtés demeurent.

En ayant effectué des recherches sur la fréquence de ces trois mots à partir du site Legifrance, nous avons constaté que le mot rémunération est le plus utilisé dans le Code du travail, avec 589 occurrences contre 410 pour salaire et trois seulement pour rétribution.

Le terme rémunération, dérivé du latin remuneratio ${ }^{1}$ - la récompense, la rémunération -, désigne le prix pour un travail ou un service fourni. Le mot qui figure dèsle Moyen-âge dans le règlement des corporations, reste fréquemment utilisé dans les textesjuridiques : aussi bien dans le Code de commerce qui évoque par exemple " la rémunération des dirigeants sociaux », que dans le Code du travail, qui précise à l'article L. 3221-3 que « la rémunération du salarié comprend le salaire ou traitement ordinaire de base ainsi que tous les avantages et accessoires payés par l'employeur au salarié ». Si nous nous en tenons à cette définition, nous pouvons constater le terme rémunérationrenvoie, ainsi que nous l'avons noté, à une notion beaucoup plus large que celle de salaire. Néanmoins, le Code du travail présente une certaine ambiguïté, de nombreux articles employant la formule «telle rémunération ne fait pas partie du salaire » ce qui sous-entendrait que le mot rémunération renvoie exclusivement aux accessoires. Certains juristes se sont émus de cette imprécision et réclament une « recodification à droit constant » (c'est-à-dire que l'on précise la terminologie sans changer le droit). (Auzero, 2014: 929)

Le mot rétribution, du latin ecclésiastiqueretributio-la récompense (céleste) -, peut aussi être considéré comme un synonyme de rémunération. Il a cependant presque disparu aujourd'hui du vocabulaire juridique alors qu'il figurait à plusieurs reprises dans la première édition du Code civil en 1804, le salaire étant par exemple défini comme « la rétribution que paye le maître à l'ouvrier » (Code civil des Français, 1804, article 1825).Même si le mot a conservé le sens de ce qu'on gagne par son travail, il est davantage utilisé dans son sens étymologique : la récompense ou la punition qui échoit à quelqu'un en contrepartie de ses actes. Aujourd'hui, dans le code du travail, il n'est plus utilisé que pour désigner la somme que verse un salarié à un intermédiaire (par exemple à un agent artistique dans l'industrie du spectacle) pour obtenir un emploi. (p.e. Code du travail, article L7121-13)

Salaireest dérivé du mot latin salarium qui désignait au début de la Rome républicaine, à une époque où la monnaie n'existait pas encore, la ration de sel que

${ }^{1}$ Toutes les définitions des mots latins viennent des dictionnaires GAFFIOT, LatinFrançais (1934) et GOELZER, Latin-Français (1893) 
recevaient les soldats en période de campagne. Le sens du mot a glissé par la suite pour désigner toute forme de rémunération accordée contre un travail. C'est dans ce sens que le mot est employé à de nombreuses reprises par le Code Théodose et le Code Justinien (p.e. Codex Iustinianus, Institutes, liber I, vol. 1, § 476). Le terme est cependant concurrencé par d'autres expressions comme stipendium ou dieta, dont les dérivés en français ont pris des sens différents.Le terme salarium, francisé en salaire, figure dès le XII ${ }^{\mathrm{e}}$ siècle dans les règlements des corporations ou dans les coutumes municipales (par exemple celles de Beauvais et d'Amiens).

A la fin du XVIII ${ }^{\mathrm{e}}$ siècle, avec les prodromes de la révolution industrielle, le mot prend le sens que nous lui connaissons aujourd'hui. Du point de vue juridique, le salaire apparaît comme la contrepartie du travail accompli dans le cadre d'un contrat de travail ou de louage de services pour reprendre la terminologie qui figure dans l'édition de 1804 du Code civil.Si l'édition actuelle du Code du travail ne donne pas de définition du salaire, la chambre sociale de la Cour de cassation considère qu'est salaire toute rémunération d'un travailleur en état de subordination (Cour de cassation, chambre sociale, 10 octobre 1979). Il convient de remarquer que dans le droit français la conséquence principale du contrat de travail est d'établir un lien de subordination entre le salarié et l'employeur.

Jusqu'au début du $\mathrm{XX}^{\mathrm{e}}$ siècle, l'obligation de verser un salaire a été étroitement liée à l'accomplissement du travail. Toute la jurisprudence de l'époque va dans ce sens, en affirmant que si le travail n'est pas fourni, le salaire n'est pas dû. Dans l'entre-deux-guerres, puis surtout après la Libération, ce lien entre versement du salaire et accomplissement du travail s'est distendu. En effet, des sommes versées hors de toute prestation effective de travail sont désormais assimilées au salaire: indemnités de congés payés, indemnisation compensatrice en cas de maladie(à ne pas confondre avecl'indemnité journalière en cas d'arrêt de travail pour cause de maladie versée par la Sécurité sociale), heures de délégation payées aux délégués du personnel et aux représentants syndicaux, rémunération des temps de pause, pour les ouvriers qui travaillent à la chaîne, et mêmesalaire d'inactivité, en cas de chômage technique. Pour désigner l'ensemble de ces versements, on emploie souvent l'expression : salaire social.

La loi dispose que le contrat de travail doit stipuler le montant, la nature et les modalités de calcul du salaire. En ce qui concerne ce dernier point, le salaire peut être calculé au temps (à l'heurepour les ouvriers, les techniciens et les employés, au moispour les cadres, indépendamment du nombre d'heures effectuées) ou $a u$ rendement (synonyme : salaire à la tâche) : pour les ouvriers, on parle de salaire aux pièces; pour les commerciaux de commission; pour le personnel de restauration de service).

Si le montant du salaire est, théoriquement, librement négocié par les parties, il ne saurait cependant être inférieur au salaire minimum fixé par un décret du gouvernement. Ce salaire minimum a été désigné successivement par les siglesS.M.I.G. (salaire minimum interprofessionnel garanti), remplacé en 1970 par S.M.I.C. (salaire minimum interprofessionnel de croissance). Les expressions familières et journalistiques, smigard puis smicard (formées à partir du sigle et du suffixe -ard qui possède en général un sens péjoratif), nomment les personnes payées au salaire minimum. 
Du point de vue comptable, il convient de distinguer : le coût salarial (la rémunération du salarié + les cotisations salariales + les cotisations patronales $)^{1}$, le salaire brut (la rémunération du salarié + les cotisations salariales + éventuellement certaines allocations sociales) et le salaire net (le salaire brut - les cotisations sociales). Rappelons qu'en France, le montant de l'impôt sur le revenu des personnes physiques n'est pas retenu sur le salaire. Le salarié touche donc le salaire net imposable qu'il doit déclarer au fisc. Ce salaire net imposable est supérieur au salaire net à payer- la somme que verse effectivement l'employeur à son salarié -car les montants de certaines cotisations sociales, déduites du salaire brut, sont néanmoins imposables au titre de l'impôt sur le revenu.

\section{Les accessoires du salaire}

Le salarié, outre le salaire de base dont le montant est fixé par le contrat de travail, bénéficie généralement de rémunérations supplémentaires soit en nature, soit en espèces, que l'on désigne parfois sous le nom de salaire indirect. Dans le premier cas, on parle d'avantages, et dans le second d'accessoires. Nous passerons rapidement sur les avantages en nature, qui ont joué un rôle essentiel jusqu'au milieu $\mathrm{du} \mathrm{XX}^{\mathrm{e}}$ siècle, à une époque où la France était d'abord un pays rural.Il convient de noter que les avantages en naturene sont pris en compte dans la rémunération du salarié que lorsque ce dernier peut en faire un usage à titre privé. Ainsi, les fournitures de vêtements de travail, d'outils, de matériel informatique, etc. ne sont-elles pas considérées comme des avantages en nature.

En ce qui concerne les véhicules, il existe dans le Code du travail une distinction entre ceux qui sont de service(ou de société), qui ne peuvent être utilisés que dans le cadre professionnel et ne sont donc pas considérés comme des avantages ; et ceux de fonction que le bénéficiaire peut aussi utiliser à titre privé, les week-ends ou pendant les vacances. Cette distinction entre véhicules de service et véhicules de fonction, qui a un caractère souvent artificiel, fait l'objet d'un contentieux très important, en ce qui concerne par exemple, le paiement des amendes, l'entretien du véhicule, la prise en compte du véhicule de fonction dans le calcul des indemnités de licenciement, les trajets entre le domicile et le lieu de travail avec un véhicule de service, etc.

Les accessoires désignent les sommes d'argent que le salarié touche en plus de son salaire. Dans ce domaine, le vocabulaire présente une certaine imprécision. Dans les contrats de travail, les textes réglementaires ou les bulletins de salaire figurent des expressions comme primes, indemnités, gratifications, treizième mois, étrennes(versées à l'occasion de la nouvelle année, du latin strena - présents qu'on fait un jour de fête pour servir de bon présage), etc. qui ne sont souvent pas véritablement définis. De plus chaque entreprise a tendance à utiliser son propre jargon, ce qui rend les choses encore plus compliquées.

Sur le plan juridique, il convient de distinguer d'une part les accessoires qui sont garantis par le contrat de travail et qui ont un caractère obligatoire (prime d'ancienneté, prime de rendement, prime d'objectif, prime de pénibilité, etc.) et d'autre part, ceux qui dépendent de la libéralité du chef d'entreprise et qui ont un caractère facultatif (treizième mois, gratification à l'occasion d'un évènement relevant

${ }^{1}$ On rencontre aussi parfois l'expression salaire super-brutavec le même sens. Nous déconseillons cependant son emploi car contrairement à coût salarial, elle ne figure ni dans la loi, ni dans les textes réglementaires, ni sur les bulletins de salaires. 
de la vie personnelle du salarié comme un mariage ou une naissance, prime de fin d'année, prime exceptionnelle, etc.).

Il existe aussi une différence entre les accessoires qui rentrent dans la rémunération du salarié et de ce fait sont imposables, et les indemnités (Code civil, article 1135) qui servent à rembourser des dépenses liées à l'activité professionnelles, et ne sont pas considérées pour cette raison comme faisant partie de la rémunération du salarié. Le caractère imprécis du vocabulaire multiplie les pièges. Par exemple "l'indemnité de congés payés" ou "l'indemnité de départ en retraite " sont considérées comme des accessoires du salaire, alors que la "prime de panier » (ou «prime de repas ») versée aux salariés qui sont en déplacement professionnelest classée comme une indemnité.

Les salariés en contact direct avec la clientèle dans certains secteurs professionnels (hôtellerie, restauration, spectacles, salons de coiffure, tourisme...) peuvent aussi bénéficier d'un pourboire (pourliche en argot) qui est défini comme une gratificationqu'un client laisse au personnel en supplément du service fourni. Le terme apparu au XVII ${ }^{\mathrm{e}}$ siècleest dérivé d'une expression utilisée autrefoislorsqu'on donnait une récompense à quelqu'un pour le remercier : "Tenez, pour boire à ma santé ! ».Le code du travail (article L. 3244-1) oblige les employeurs à reverser l'intégralité des pourboires à leurs salariés. Il leur est en effet strictement interdit d'opérer des retenues. Depuis une loi de 1933, la pratique dite de «l'achat du tablier» qui autorisait l'employeur à pratiquer des retenues sur les pourboires pour compenser la fourniture de vêtements professionnels est prohibée.

Toute heure de travail accomplie, à la demande de l'employeur, au-delà de la durée légale de 35 heures constitue une heure supplémentaire (heure sup dans la langue familière). Les heures supplémentaires ouvrent droit à une rémunération plus favorable (taux horaire majoré). La loi ne prévoit pas de règles en matière de salaire pour les dimanches et jours fériés travaillés. Ces règles dépendent entièrement des conventions collectives, nombre d'entre-elles prévoyant ainsi des majorations de salaires ou des primes (prime de dimanche). En ce qui concerne le travail de nuit, entre $21 \mathrm{~h}$ et $6 \mathrm{~h}$, il faut travailler au moins deux fois par semaine trois heures d'affilée dans cette tranche horaire pour bénéficier du statut de travailleur de nuit. Ces heures peuvent faire l'objet soit d'une majoration, soit d'un repos compensateur. En outre, le salarié peut bénéficier d'une prime de nuit.

L'un des grands projets du général de Gaulle consistait en l'association du capital et du travail. Dès 1959, le gouvernement Debré, légiférant par ordonnance, introduit le principe de l'intéressement des salariés aux bénéfices. Une loi de 1967 institue une épargne salariale obligatoire pour toutes les entreprises de plus de 50 salariés. Il s'agit de redistribuer aux salariés une partie du bénéfice. Dans le cadre de cette loi, quatre dispositifs ont été mis en place : le plan épargne entreprise (PEE), le plan d'épargne interentreprises (PEI), le plan d'épargne pour la retraite collectif(PERCO), la prime d'intéressement.Sans remettre en cause les dispositifs existants, la législation a évolué ces dernières années avec pour objectif de développer l'actionnariat salarié. D'après les dispositions récentes (loi de 2008 sur l'actionnariat salarié), une part du capital des sociétés cotées en Bourse doit être versée sur le PEE. D'autre part, les salariés en cas d'augmentation du capital doivent bénéficier d'une offre réservée aux salariés(ORS).

Sans faire partie directement du salaire, les ouvres sociales peuvent être aussi considérées comme des avantages accordés aux salariés. Sous ce terme, on désigne les activités sociales (par exemple, les " pots » de départ en retraite ou les colonies de vacances pour les enfants du personnel), culturelles et sportives établies dans l'entreprise au profit des salariés ou de leurs familles. Le comité d'entreprise assure normalement leur gestion.

XLinguae Journal, Volume 9 Issue 2, April 2016, ISSN 1337-8384 


\section{III - Les termes professionnels}

Une des spécificités du vocabulaire des rémunérations en français réside dans la présence d'un grands nombre de termes propres à une profession donnée. Cette terminologie concerne aussi bien le salaire de base que les accessoires. Sans vouloir viser àl'exhaustivité, nous avons aussiinclus des expressions disparues de l'usage courant. Pour plus de clarté, nous avons classé les expressions par profession.

Aucune étude approfondie de ce vocabulaire n'ayant été entreprise, nous avons consulté systématiquement les ouvrages suivants : Trésor de la Langue française, Dictionnaire d'Emile Littré en 4 volumes (1873-1877), Rey (2010), Cornu (2013), Guinchard (2014), Colin (2010), les codes de commerce, des impôts, de la Sécurité sociale et du travail dans les versions accessibles sur legifrance.gouv.fr.

A. Les professions libérales : Pour désigner la rétribution d'un membre d'une profession libérale, on utilise généralement le mot honorairesdérivé du latinhonorarium qui signifie rétribution d'une charge, cadeau. Le mot est apparu en français à la fin du XVI ${ }^{\mathrm{e}}$ siècle. Il est utilisé habituellement au pluriel, même si on peut parfois le rencontrer au singulier (en général dans des textes littéraires, très rarement dans des documents professionnels).

1. Les avocats : les honoraires de l'avocat sont librement établis. Les règles déontologiques imposent néanmoins que les honoraires soient fixés en tenant compte de la difficulté de l'affaire, de l'usage, de la situation financière du client, des frais exposés par l'avocat, de sa notoriété, etc. Il existe quatre façons de calculer les honoraires: leshonoraires au temps passéqui résultent à la fin du dossier d'une simple multiplication du temps passé par le taux horaire déterminé à l'origine ; leshonoraires forfaitairesdans ce cas, l'avocat convient avec son client d'une rémunération globale, non modifiable; les honoraires de résultat - une part des honoraires est calculée en proportion des résultats financiers obtenus par l'avocat; l'abonnement, lorsque le client recourt périodiquement et régulièrement à son avocat, il est possible de conclure avec lui un contrat d'abonnement, en général annuel.Habituellement, le client signe, lorsqu'il fait appel à un avocat, une convention d'honorairesqui stipule la façon dont ceux-ci seront calculés Avant de commencer à travailler sur un dossier, l'avocat demande normalement le versement d'une provision qui représente une avance sur les honoraires. Outre ces derniers, le client doit aussi payer les débours(expression dérivée du verbe débourser) qui désignent les avances que font les avocats pour des dépenses qui ne sont pas incluses dans les honoraires comme les frais de copie, le coût de délivrance d'actes, les frais de transport ou de correspondance, la rémunération de tierces personnes, les frais de publicité légale, etc.

2. Les médecins : Il convient de distinguer d'un côté les médecins hospitaliers qui sont des salariés payés mensuellement, indépendamment du nombre d'actes effectués, et d'autre part les médecins libéraux qui sont assimilés à des travailleurs indépendants. Les médecins libéraux sont normalement payés à l'acte : pour chaque consultation ou soin dispensé, ils touchent des honoraires. Ces derniers sont théoriquement libres. Néanmoins la plupart des médecins ont signé une convention avec l'assurance maladie fixant les tarifs des consultations et des actes médicaux, mais qui les autorise dans certains cas à pratiquer des dépassements d'honoraires qui ne seront pas remboursés au patient. Un débat se déroule à l'heure où nous écrivons cet 
article pour introduire le paiement (comme c'est le cas en République tchèque) par capitation - du latin caput, la tête : il s'agit d'une rémunération annuelle versée à un médecin pour prendre soin d'un patient inscrit à son cabinet pour une période donnée, par exemple un an. La rémunération du médecin est forfaitaire et versée que le malade consulte une ou plusieurs fois ou non. Le niveau de rémunération pour chaque inscription est adapté aux caractéristiques cliniques et à l'âge des malades.

3. Les officiers ministériels (notaires, huissiers, commissaires-priseurs, etc.) : Outre le terme honoraires, on emploie souvent le terme émoluments du latin emolumentum - somme payée à un meunier pour moudre son grain - pour désigner la rémunération des officiers ministériels. Apparu au XIII ${ }^{\mathrm{e}}$ siècle, le mot au départ était utilisé pour désigner le salaire versé par le roi à ses agents. Au cours des siècles, le sens du mot s'est progressivement réduit pour ne plus désigner que la rémunération des officiers ministériels.Fixés par l'autorité publique, les émoluments sont proportionnels ou fixes en fonction de la nature de l'acte. Dans ce sens, le mot doit toujours être employé au pluriel, car le terme au singulier désigne la part d'actif hérité par un légataire. Attesté jusqu'à la fin du XVIII ${ }^{\mathrm{e}}$ siècle, le verbe émolumenter, dans le sens de verser des émoluments a disparu de l'usage. Le terme " émoluments » qui appartient à un registre de langue spécialisée est souvent ignoré du citoyen ordinaire qui préfère utiliser l'expression frais de notaire. Ce terme comprend non seulement les émoluments mais aussi les taxes et droits perçus par les notaires pour le compte de l'administration fiscale.

B. Les artistes et littérateurs : Au cours des siècles le vocabulaire a beaucoup fluctué pour désigner les rémunérations des auteurs et des professions artistiques. De fait, sous l'Ancien régime, un artiste, pour vivre, avait besoin d'un mécène, le plus prestigieux d'entre eux étant bien sûr le roi, qui lui versait une pension - du latin pensio, indemnité. Depuis la Révolution, cette pratique est devenue marginale. Si le terme "pension» a disparu, on continue cependant à employer dans certains cas celui de pensionnaire pour désigner un artiste qui bénéficie d'une rente versée par l'Etat: un pensionnaire de la Comédie-Française, de la Villa Médicis, etc.

1. Les auteurs : Lesdroits d'auteur sont définis comme la redevance, variable selon les cas, perçue par un auteur lors de la publication par un éditeur, l'adaptation, la traduction, la représentation de son œuvre. L'expression doit toujours être employée au pluriel. Au singulier, elle désigne la propriété littéraire ou artistique. Dans un contrat d'édition, les droits d'auteur représentent un pourcentage sur les ventes. L'usage veut que l'éditeur verse un à-valoirà l'auteur lors de la remise du manuscrit. Cettesomme est définitivement acquise, même si les droits d'auteur se révèlent d'un montant total plus faible que l'à-valoir. Celui-ci sera déduit des droits d'auteur si ces derniers sont plus élevés que l'à-valoir. Si un ouvrage est publié à compte d'auteur (on emploie aussi le mot autoédition, en particulier pour une œuvre publiée en ligne), les revenus tirés de la vente du livre sont considérés comme des bénéfices.

2. L'industrie du spectacle : Le terme cachet (cachetonen argot - ce dernier terme, par extension, a aussi le sens du prix de la passe, de la relation sexuelle tarifée, souvenons-nous des liens étroits entretenus aux XVIII ${ }^{\mathrm{e}}$ et $\mathrm{XIX}^{\mathrm{e}}$ siècles entre le monde des théâtres et celui de la prostitution, cf. Illusions perdues, La dame aux caméliaset Nana)désigne la rétribution d'un 
artiste, d'un acteur, d'un musicien, etc. par représentation ou par concert.Apparue au tout début du XVII ${ }^{\mathrm{e}}$ siècle, l'expression désignait à l'origine la carte qu'un élève remettait à son professeur pour qu'il y mette son cachet afin de décompter les leçons. Par métonymie le terme a fini par désigner les leçons particulières, puis par analogie la rémunération des artistes. Le verbe cachetonner a le sens péjoratif de multiplier les engagements sans tenir compte de la valeur artistique des œuvres interprétées.

3. Les journalistes : Quand un journaliste est rémunéré en fonction de la longueur de son article (nombre de signes ou de lignes), on dit qu'il est payé à la pige (le terme est utilisé dans les articles L. 761-1 et L. 761-2 du Code du travail.). L'expression est aussi utilisée pour désigner la rémunération d'un collaborateur régulier d'une émission de radio ou de télévision qui n'a pas un statut de salarié. Le terme «pige» vient du vocabulaire des typographes: il désignait le nombre de signes qu'un compositeur devait fournir à l'heure.

C. Les salariés : Il existe un grand nombre de synonymes de salaire. Si certains relèvent de la langue standard ou juridique, beaucoup appartiennent à la langue populaire.

1. Les ouvriers : Dans le monde ouvrier, les expressions paye(autre graphie, paie) oumoisont été jusqu'à la fin des années 1960 d'un usage bien plus courant que salaire. Si ce dernier mot a aujourd'hui complètement supplanté les deux autres termes, ceux-ci continuent à être utilisés dans des expressions figées comme bulletin de paye, livre de paye, jour de payeoutreizième mois, toucher son mois. Il a existé bien sûr de nombreux termes familiers ou argotiques pour désigner le salaire, mais tous ont disparu. Nous ne citerons que la douillance - du verbe douiller, en argot, payer - que l'on trouve en particulier chez L.-F. Céline : «C'était un drôle de boulot, assez marrant, faut bien le dire, mais pour la douillance assez terne, pas très généreux. Pas de quoi se régaler du tout », Bagatelles pour un massacre, 1937. Jusqu'au milieu du $\mathrm{XIX}^{\mathrm{e}}$ siècle, à une époque où le terme contrat de louage était plus utilisé que celui de contrat de travail, le terme loyera été couramment utilisé comme synonyme de salaire, de rétribution d'un travail. Le mot figure déjà avec ce sens dans la Chanson de Roland (vers 2584 : «Ki mult te sert, malvais luer l'en dunes»= à celui qui te sert beaucoup, tu donnes un mauvais loyer). Cette acceptation a aujourd'hui complètement disparu.

2. Les employés : Un terme est spécifiquement utilisés pour désigner le salaire versé aux employés (et plus particulièrement aux vendeurs) celui d'appointements. Le terme au Moyen-âge était utilisé au singulier pour désigner un arrangement financier entre les parties dans le but de mettre fin à un procès. Si dès le $\mathrm{XVI}^{\mathrm{e}}$ siècle, on relève les premières attestations du mot dans le sens de salaire ou de rétribution, il faut attendre cependant le $\mathrm{XIX}^{\mathrm{e}}$ siècle pour que l'expression se banalise et entre dans les dictionnaires. Pour les commerciaux, la rétribution est calculée partiellement ou totalement au pourcentage. On parle decommissionou de boni (pluriel des bonis) de l'expression latine aliquid boni- quelque chose de bon - pour les représentants, de guelte (dérivée de l'allemand das Geld - l'argent) pour les vendeurs de boutiques de mode. Pour les domestiques, on employait jusqu'au début du $\mathrm{XX}^{\mathrm{e}}$ siècle le terme de gages (du francique waddi latinisé en waddium - ce qu'on met ou laisse en dépôt, comme garantie d'une dette, 
de l'exécution de quelque chose). Le mot n'est plus guère utilisé aujourd'hui que de manière figurée (et en général, dans un sens péjoratif) : être aux gages de quelqu'un- être au service de quelqu'un.

3. Les dirigeants de sociétés : En ce qui concerne les gérants de SARL, ils sont considérés comme des travailleurs indépendants s'ils possèdent plus de $50 \%$ du capital de la société qu'ils administrent. Ils ne touchent donc pas de salaire mais des bénéfices. En cas contraire, ils ont le statut de salarié. Pour désigner la somme qu'ils touchent, on emploie cependant plutôt le terme de rémunération que celui de salaire. Celle-ci est complétée généralement par une prime d'intéressement - un pourcentage des bénéfices.

Les dirigeants des sociétés de capitaux (SA et SAS) ont toujours le statut de salariés. Leur rémunération peut être fixe ou variable(en fonction du chiffre d'affaires, de la valorisation de la société si elle est cotée en Bourse, ou des bénéfices réalisés). Également intitulés plan d'options sur actions, les stockoptions sont une des modalités les plus courantes pour rémunérer les dirigeants des grandes sociétés. Ce système recouvre deux variantes : les options de souscription d'actions et les options d'achat d'actions. L'option d'achat d'actions consiste pour une société par actions à donner aux bénéficiaires le droit d'acheter des actions existantes de la société à un prix fixé au jour où l'option est consentie par la société, et ce dans le délai qu'elle détermine. L'option de souscription d'actions consiste pour une société par actions, qui projette de procéder à une augmentation de capital, de donner aux bénéficiaires le droit d'acheter les futures actions, dans un délai donné et à un prix déterminé. L'intérêt de ces mécanismes réside essentiellement dans le fait que le prix d'achat convenu est inférieur au prix de marché.

Le parachute doré (ou en or) - terme journalistique - est une prime de départ. Il s'agit d'une somme forfaitaire qui est automatiquement versée au dirigeant au moment de la rupture de son contrat, quel qu'en soit le motif. En cas de départ à la retraite, le dirigeant d'une grande société peut également recevoir une retraite chapeau - terme journalistique - également appelée retraite supplémentaire à prestations définies ou retraite surcomplémentaire. Il s'agit d'une rente dont le montant est fixé à l'avance et qui est versée à l'ancien dirigeant jusqu'à son décès.

Les administrateurs de société (membres des conseils de surveillance et d'administration) touchent des jetons de présence calculés en fonction de l'assiduité aux réunions auxquelles ils assistent.

4. Les fonctionnaires et autres agents de l'Etat: Depuis l'époque de Napoléon $\mathrm{I}^{\mathrm{er}}$, le terme traitementqui est défini dans le droit administratif comme la rémunération principale des fonctionnaires publics, civils et militaires, payable par douzième mensuel et variable(La semaine juridique, 2009-4) aremplacé les diverses expressions souvent fleuries utilisées sous l'Ancien régime. Rappelons qu'avant la Révolution, il existait deux types d'agents du roi : les officiers qui étaient détenteurs d'une charge achetée au roi, qu'ils pouvaient revendre ou transmettre à leurs héritiers; et les commissaires, qui étaient nommés et révoqués par le roi. Les premiers ne touchaient pas la plupart du temps de rémunération, mais faisaient payer leurs services aux administrés : par exemple les juges réclamaient aux justiciables le versement d'épices que le dictionnaire de Furetière (1690) définit comme les droits que les juges de certains tribunaux sont autorisés à faire payer aux parties pour la visite des procès par écrit ; les agents du fisc touchaient un pourcentage sur les amendes infligées ou les impôts prélevés,

XLinguae Journal, Volume 9 Issue 2, April 2016, ISSN 1337-8384 
qu'on appelait le casuel, ou comme le sont toujours les notaires, les officiers étaient rémunérés à l'acte (émoluments). Les commissaires, en particulier les ambassadeurs ou les intendants (qui apparaissent comme les précurseurs des préfets), bénéficiaient de gratifications ou de pensions versées par le roi, mais comme il leur faillaient subvenir à toutes les dépenses de la charge (en particulier la rémunération des agents subalternes), ils devaient disposer d'une fortune personnelle considérable. Le duc de Saint-Simon, comme on sait, s'est ruiné lors de son ambassade à Madrid. Les agents subalternes (commis, secrétaires, etc.) touchaient des gages, ce qui les ravalaient au rang de domestiques.

Outre leur traitement, les fonctionnaires touchent de nombreuses primes et indemnités. Nous pouvons citer : la prime de fonction et de résultats (pour les fonctionnaires d'autorité) ; la prime de sujétion(destinée à compenser des conditions particulières de travail, par exemple pour les policiers avoir des périodes d'astreinte, c'est-à-dire de disponibilité en dehors de leurs heures de service) ; l'indemnité de résidence ; l'indemnité de mobilité ; la prime de langue(pour les personnels utilisant une langue étrangère pour l'exécution de leur service); le supplément pour charge familiale(la majorité des fonctionnaire étant soumis au principe de mobilité, il est prévu une indemnité spéciale pour compenser les désagréments que cela entraîne pour la famille), etc.

Pour les militaires, le terme la solde (dérivé de l'italien soldo - même sens qu'en français - lui-même venant du latin solidus - le sou) est préféré à celui de traitement. On parle de lasolde à l'air ou en mer pour désigner les indemnités supplémentaires versées aux aviateurs et parachutistes pour des missions en vol, ou aux marins lorsqu'ils naviguent. L'expression solde de guerrea été remplacée en 1975 par prime opérationnelle. Jusqu'en 1945, les hommes du rang - simples soldats, soldats de première classe et caporaux ne touchaient pas une solde mais un prêt, terme défini par Littré (1877) comme « la somme allouée par l'État pour l'entretien du soldat».

En ce qui concerne les élus, on parle d'indemnités parlementaires pour les députés et les sénateurs. Les ministres et secrétaires d'État touchent un traitement auquel s'ajoute une indemnité de fonction. Le Président de la République, outre un traitement et une indemnité de fonction, dispose d'une dotation annuelle pour couvrir certains frais inhérents à sa charge (cadeaux offerts aux chefs d'État étrangers, frais de représentation, etc.).

D. Le clergé : La terminologie concernant la rémunération des ecclésiastiques a beaucoup changé suivant les périodes. Sans remonter jusqu'au Moyenâge, nous nous limiterons à évoquer la période de l'Ancien régime. La dîme, du latin decima - dixième - était la principale source de revenu de l'Église. Il s'agissait à l'origine d'un impôt payé en nature sur les récoltes et le bétail, mais dès la fin du Moyen-âge, il avait été remplacé par une somme d'argent. Contrairement à ce que laisse entendre l'étymologie du mot, le prélèvement était très inférieur au dixième des revenus. En France, à la veille de la Révolution, il s'établissait suivant les provinces entre $1 / 15^{\mathrm{e}}$ et $1 / 30^{\mathrm{e}}$ des revenus disponibles. Normalement, un partage était effectué dans chaque paroisse, entre l'évêque qui touchait un tiers de la dîme, et le curé de la paroisse qui en conservait les deux tiers. Néanmoins, il arrivait assez souvent que le curé titulaire ne résidât pas dans sa paroisse et qu'il se déchargeât de ses fonctions sur un desservant qui devait se contenter de la portion congrue, c'est-à-dire de la part (misérable) de la dîme que lui 
laissait le curé titulaire. L'expression est restée utilisée jusqu'à nos jours pour désigner une rémunération insuffisante : toucher la portion congrue, être réduit à la portion congrue.Avec le Concordat de 1801, le clergé fut fonctionnarisé et reçut un traitement. Les articles organiques du Concordat étendirent cet avantage aux pasteurs protestants ainsi qu'aux rabbins, et plus tard même aux imâms dans les départements d'Algérie. Les lois de 1905 sur la séparation de l'Eglise et de l'Etat mirent fin à cette situation. Depuis cette date, ce sont les fidèles qui entretiennent le clergé en versant le denier $\boldsymbol{d u}$ culte(ou du clergé).Les ecclésiastiques touchent en outreles offrandes des fidèles à l'occasion de certainescérémonies (baptêmes, mariages, messes à la mémoire des défunts, etc.) qu'on appelle le casuel.

\section{Lexique des rémunérations en français et leurs équivalents tchèques}

Le lexique des "rémunérations »(odměňování) en français est très riche, tandis que le tchèque juridique distingue trois termes clés (mzda-plat-odměna) en fonction du type de contrat de travail (CDD, CDI pour une activité salariée dans le secteur privé $(m z d a)$, public (plat) ou pour certains types de CTT exercés en dehors de l'activité salariée principale ou même comme une activité indépendante (odměna). Le champ lexical français est beaucoup plus étendu comme nous avons pu le constater. En effet, il comprend des termes qui changent en fonction de l'activité professionnelle exercée. Le terme même de la rémunération est polysémique, parce qu'en dehors du sens générique large il possède aussi des sens plus spécialisés, étant le synonyme de salaire -avec ou sans accessoires, partie variable du salaire (platy a odmény).

\begin{tabular}{|c|c|}
\hline RÉMUNÉRATIONS & ODMĚŇOVÁNÍ \\
\hline rémunération $f$ & odměňování; plat; plat a odměna \\
\hline$\sim$ des salariés & platy a odměny zaměstnanců \\
\hline$\sim$ des dirigeants de sociétés & platy členů vedení obchodních společností \\
\hline$\sim$ des temps de pause & placené přestávky/pauzy v práci \\
\hline$p l$ (employés) & platy zaměstnanců ve službách ve F \\
\hline bénéfices $m p l$ & zisk (z přijmů komerčních i nekomečních) \\
\hline cachet $m$ & $\begin{array}{l}\text { odměna /honorár umělce, herce, } \\
\text { hudebníka ve } \mathrm{F}\end{array}$ \\
\hline casuel $m$ & štóla (poplatek kněžím) \\
\hline commission $f$ & $\begin{array}{l}\text { provize (podílová odměna) obchodních } \\
\text { zástupců ve } \mathrm{F}\end{array}$ \\
\hline denier $m$ du culte & $\begin{array}{l}\text { příspěvek na bohoslužbu (finanční } \\
\text { náhradakněží) } \mathrm{F}\end{array}$ \\
\hline droits $m p l$ d'auteur & $\begin{array}{l}\text { autorský honorár } \quad \text { (umělecká } \\
\text { intelektuální tvorba), autorská práva }\end{array}$ \\
\hline - à-valoir & $\begin{array}{l}\text { - záloha na honorář (po odevzdání } \\
\text { rukopisu) }\end{array}$ \\
\hline - bénéfices & $\begin{array}{ll}\text { - } \quad \text { ziskz výnosů } \\
\text { právaautorského,tantiémy (umělci)Č }\end{array}$ \\
\hline émoluments $m p l$ (officiers ministériels) & $\begin{array}{l}\text { odměny a náhrady vyšších úředníků ve } \mathrm{F} \\
\text { (i soudních) }\end{array}$ \\
\hline étrennes $f p l$ & $\begin{array}{l}\text { provize, jednorázová odměna na začátku } \\
\text { roku (popeláŕi, kominící ...) }\end{array}$ \\
\hline guelte $f$ & $\begin{array}{l}\text { provize prodavačů za prodané zboží } \\
\text { (módní obchody) }\end{array}$ \\
\hline honoraires $m p l$ (professions libérales) & $\begin{array}{l}\text { odměny lékařú, odměny a náhrady } \\
\text { advokátů,... }\end{array}$ \\
\hline
\end{tabular}


$\sim$ au résultat

$\sim$ au temps passé

$\sim$ conventionnés

être payé à l'acte x par capitation convention d'honoraires

$\sim$ forfaitaires

déboursmpl

provision $f$

indemnité $f$ parlementaire

jetons $m p l$ de présence

pige $f$ (être payé à la pige $\rightarrow$ pigiste)

salaire $m$

service $m$ (x pourboire)

solde $f$

traitement $m$ (fonctionnaires)
- $\quad$ podle dosažených výsledků

(advokáti)

- $\quad$ za ztrátu času/za promeškaný čas

(advokáti)

- $\quad$ smluvní odměna lékaře s

pojišt'ovnou (konzultace a výkony)

odměna lékaře podle výkonu (F)x

kapitační platba ( $\breve{C})$

smluvní odměna advokáta

- paušální odměna advokátů (podle tarifu)

záloha na výlohy (advokáta, vyšších úředníků)

paušální jednorázová záloha advokátovi na výdaje (F)

poslanecký plat/ poslanecká náhrada ve F

odměna za práci $\mathrm{v}$ dozorčí radě $\mathrm{a}$ představenstvu a.s. (F) ; tantiémy (Č)

odměna/honorář nezávislého novináře, externistu (od řádku, od stránky)ve F ( $\rightarrow$ nezávislý novinář)

mzda, plat

provize číšníků (součást platu)ve $\mathrm{F}$ x (spropitné)

plat vojáků, žold

plat státních zaměstnanců ve $\mathrm{F}$

En ce qui concerne le «salaire», il existe des points similaires entre le français et le tchèque au niveau des modalités ou types de salaire qui n'est pas toujours forcément lié au travail fourni (náhrady $m z d y$ ), mais il y a des différences surtout au niveau des accessoires (doplňky, pohyblivá složka mzdy) où la terminologie est très variée et souvent ambiguë en raison de la polysémie des termes dans les deux langues (indemnités, primes,gratifications / príplatky, osobni ohodnoceni, prémie, odmény). À cause de cette imprécision de la terminologie, certains termes n'ont pas d'équivalents uniques, mais leur sens change en fonction du contexte linguistique (collocations) et juridique.

SALAIRE $m$

$\sim$ au pourcentage /à la commission

$\sim$ au rendement / à la tâche

$\sim$ au temps (à l'heure/ au mois)

$\sim$ aux pièces

$\sim$ brut / super-brut / coût salarial

$\sim$ d'inactivité /social (chômage technique)

$\sim$ de base + accessoires du salaire $m p l$

$\sim$ forfaitaire

$\sim$ indirect (avantages en nature $\mathrm{x}$ en espèce

/ accessoires )

\section{MZDA, PLAT}

podílová /provizní mzda

výkonová mzda

časová mzda (hodinová /měsíční plat)

úkolová mzda

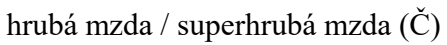

tzv. sociální mzda za dočasnou nucenou dovolenou ve $\mathrm{F}$

základní mzda (pevná složka mzdy) + doplňky mzdy (pohyblivá složka mzdy)

paušální mzda

nepřímá mzda (naturální x peněžní mzda /doplňky mzdy) 
$\sim$ minimum conventionnel

$\sim$ minimum interprofessionnel de croissance $(\mathrm{SMIC}) \rightarrow$ smicard

$\sim$ mixte

$\sim$ net à payer

$\sim$ net imposable

$\sim$ social (salaire d'inactivité )

indemnités $f p l$

$\sim$ de chômage

$\sim$ (de) congé études

$\sim$ de congé payé

$\sim$ journalières (versées par sécurité sociale)

$\sim$ complémentaires (compensatrices) aux

indemnités journalières

$\sim$ de départ à la retraite

$\sim$ de déplacement

$\sim$ de fonction

$\sim$ de licenciement

$\sim$ de mobilité (fonctionnaires, agents publics)

$\sim$ de résidence

$\sim$ de transport

$\sim$ des jours fériés

$\sim$ des temps de pause

heures $f p l$ de délégation

heures $f p l$ supplémentaires

- taux horaires majorées

- repos compensateur

prime $f$

d'ancienneté

d'intéressement (pourcentage des

bénéfices)

$\sim$ d'insalubrité

$\sim$ d'intéressement

épargne f salariale

actionnariat $m$ salarié

options de souscription/d'achatd'actions (stockoptions) zaručená mzda ( kolektivní smlouvou ve $\mathrm{F}$ )

(SMIC) minimální mzda ve F (závislá na inflaci) $\rightarrow$ osoba pobírající SMIC

smíšená mzda

mzda k výplatě $(\mathrm{F})$

zdanitelná mzda ve F (bez soc.příspěvků a záloh na daň)

tzv. sociální mzda

př́platky; náhrady výdajů (proplacení) ; náhrady mzdy/platu (za dovolenou, svátky...)

podpora $\mathrm{v}$ nezaměstnanosti

placené studijní volno

náhrada za čerpání dovolené

nemocenská dávka

př́platek zaměstnavatele

k nemocenské

odstupné při odchodu do důchodu, odchodné

cestovní náhrady, cestovné

funkční požitky (poslanci, ministři, instituce)

odstupné při propuštění

«příspěvek na mobilitu » ve F (státní zaměstnanci)

př́spěvek na bydlení

př́spěvek na dopravu, na dojíždění do zaměstnání

náhrada mzdy za práci ve svátek

placené přestávky $\mathrm{v}$ práci

náhrada za výkon funkce (odbory, zástupci zaměstnanců) ve $\mathrm{F}$

prŕíplatek za práci přesčas

- $\quad$ zvýšená hodinová sazba $(\mathrm{F})$

- $\quad$ náhradní volno za práci

př́íplatek, prémie, odměna

př́ílatek za odpracovaná léta, věrnostní prííplatek

podíl na zisku

př́ílatek za práci ve stíženém

prostředí

podíly na zisku

povinnépodnikové spoření

zaměstnanců ve $\mathrm{F}$

«akcionáři $\mathrm{Z}$ řad zaměstnanců»( $\mathrm{F})$,

zaměstnanecké akcie (Č)

opce na úpis/na nákup akcií

XLinguae Journal, Volume 9 Issue 2, April 2016, ISSN 1337-8384 


$\sim$ d'objectif
$\sim$ de danger
$\sim$ de départ
$\sim$ de dimanche
$\sim$ de fin d'année (fac.)
$\sim$ de fonction
$\sim$ de langue
$\sim$ de nuit
$\sim$ de panier/de repas
$\sim$ de pénibilité
$\sim$ de rendement /de productivité (individuel,
$\quad$ collectif)
$\sim$ de sujétion/d'astreinte

$\sim$ de vacances

$\sim$ exceptionnelle (fac.)

gratifications $f p l$ (mariage, naissance)

- abonnement téléphonique/de téléphone

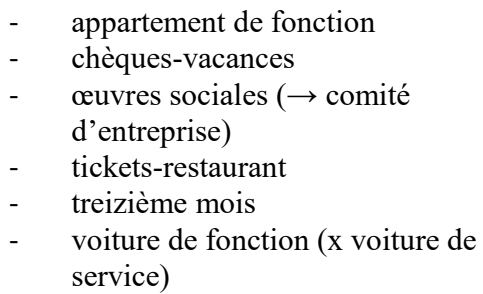

- $\quad$ pourboire $m$ prémie za splnění předem stanovených cílů

rizikový prríplatek

odstupné při odchodu z funkce (a.s.)

prríplatek za práci v neděli a ve svátek

prémie (odměna) na konci roku

příplatek za vedení

př́íplatek za cizí pracovní jazyk

příplatek za noční práci

diety, stravné (př́ílatek)

př́ílatek za ztížené pracovní prostředí

výkonnostní prríplatek

prríplatek za výkon služby mimo pracovní dobu (policisté, vojáci)ve F / príplatek za pohotovost

prríplatekna dovolenou

zvláštní př́íplatek

mimořádné odměny (jednorázové); zaměstnanecké výhody (platové a finanční benefity) $\check{C}$

firemní mobilní telefon i k soukromým účelům/bezplatné služby telefonních operátorů

služební byt

příspěvek na dovolenou

sociální benefity $(\rightarrow$ podniková rada, ve F)

stravenky

trrináctý plat

služební auto i pro soukromé účely (x jen pro služební účely)

spropitné (služby)

D'autre part, la terminologie reflète les règles juridiques d'un contexte socioculturel donné qui est en évolution. Elle comprend des termes historiquement déterminés dont certains, devenus archaïques ont disparu du langage juridique (loyer ancien synonyme de salaire - mzda, dîme - desátek, épices - odměny soudců, casuel - odměny výběrčích daní, pension - umělecká renta/sponzorský příspěvek pro umělce, gages služné,...), ont changé de sens (salaire,rémunération, casuel) ou étaient remplacés par des termes nouveaux $(S M I G \rightarrow S M I C$; rétribution $\rightarrow$ rémunération; contrat de louage $\rightarrow$ contrat de travail ; solde de guerre $\rightarrow$ prime opérationnelle,émoluments $\rightarrow$ frais de notaires, prêt $\rightarrow$ solde (žold). D'autres peuvent être tirés de la langue familière ou même argotique : parachute doré (prime de départ), retraite chapeau (retraite supplémentaire des dirigeants sociaux); êtrepayés à la pige (od rádku textu). Cependant, les termes disparus de la langue juridique persistent encore dans les expressions phraséologiques et expressions toutes faites à sens figuré : bulletin/fiche llivre de PAYE, jour de PAYE, treizième MOIS, toucher son MOIS; pensionnaire (umělec finančně podporovaný státem formou renty), congé sans SOLDE (neplacené volno), être aux GAGES de q (au service de q), toucher la portion CONGRUE (une 
rémunération insuffisante) ou dans l'argot (douillance - salaire, pourliche pourboire).

\section{Conclusion}

La rémunération qui désigne toute prestation en argent (ou en nature) fournie en contrepartie d'un travail ou d'une activité (Cornu, 2013) peut changer d'un pays à l'autre. Elle dépend des dispositions inscrites dans les codes du travail des pays respectifs. Le lexique français des rémunérationsqui varie en fonction de différentes professions et dont la richesse est historiquement déterminée, contient non seulement des termes juridiques au sens propre du terme, mais aussi des mots familiers auxquels l'usage professionnel a conféré le statut de termes spécialisés. Ils reflètent des rapports juridiques dans leurs usages spécifiques déterminés aussi bien par la loi que par la coutume et l'usage.

En comparaison avec le tchèque, le système français des rémunérationsprésente des spécificités non seulement linguistiques, mais aussi notionnelles. Il existe des instituts juridiques qui n'ont pas de correspondant dans la langue cible (p.e. le statut defonctionnaire, d'officier ministériel, épargne salariale, comité d'entreprise, salaire net imposableen français ou osobni ohodnocení, dohoda o provedení práce, dohoda opracovní činnostien tchèque) ou bien certaines notions n'y sont pas formalisées sous forme de désignants spécifiques(voiture de fonction $x$ de service, service $x$ pourboire). D'autre part, les notions identiques peuvent être désignées par des formes différentes (coût salarial - superhrubá mzda, accessoires doplňky mzdy, gratifications - mimořádné odměny), mais surtout des termes identiques (en cas de traduction littérale) peuvent avoir des significations qui non seulement ne se recouvrent pas entièrement (traitement, salaire, rémunération), mais qui peuvent différer (faux-amis : honoraires, pension, commission,...). Les expressions empruntées à l'anglais sont quasi inexistantes dansce vocabulaire. On ne les rencontre significativement que dans la terminologie des rémunérations des dirigeants des grandes entreprises (stock-options, parachute doré - calque de l'anglais golden parachute).

En fait, la traduction des termes juridiques est déterminée par une médiation linguistique et interculturelle (Gemar, 1995, Tomasek, 1998, Bocquet, 2008, Fiser, 2009). Dans le cas des différences entre les droits nationaux, la recherche des équivalences (partielles mais surtout fonctionnelles) entre les langues source et de départ doit être déterminée par la recherche des analogies produisant les mêmes effets de sens juridiques dans les deux langues ce que nous avons essayé de montrer aussi dans ce petit lexique contrastif franco-tchèque de quelques termes clés choisis.

\section{Bibliograpic references}

AUZERO, G. - DOCKES, E. 2014. Droit du travail. Paris: Dalloz. ISBN 978-2-24713009-2.

BOCQUET, C. 2008. La traduction juridique; fondement et méthode. Bruxelles: De Boeck (Collection Traducto). ISBN 978-2-8041-5928-3.

COLIN, J.-P. 2010. Dictionnaire de l'argot et du français populaire. Paris: Larousse. ISBN 978-2035852991.

CORNU, G. 2005. La linguistique juridique. Paris, Montchrestien. ISBN 2-70761425-4.

CORNU, G. 2013. Vocabulaire juridique. Paris: Presses universitaires de France. ISBN 978-2130624639.

DICTIONNAIRE d'Emile Littré en 4 volumes (1873-1877). Available online: www.gallica.bnf.fr 
FISER, Z. 2009. Preklad jako kreativní proces: Teorie a praxe funkcionalistického překládání. [Traduction comme un processus créatif. Théorie et pratique de la traduction fonctionnelle]. Brno: Host. ISBN 978-80-7294-343-2.

GEMAR, J.-C. 1995a. Traduire ou l'art d'interpreter, t. II, Langue, droit et societe: elements de jurilinguistique. Quebec: Presses de l'Universite du Quebec.

GEMAR, J.-C. 1995b. Le langage du droit au risque de la traduction. De l'universel et du particulier. In Français juridique et science du droit, Bruxelles: Bruylant.

GUILLIEN, R. - VINCENT, J. - GUINCHARD, S. et al. 2009. Lexique des termes juridiques 2010. $17^{\mathrm{e}}$ edition. Paris: Dalloz. ISBN 978-2-247-08360-2.

HESS-FALLON, B. - MAILLARD-PINON, S. - SIMON, A.-M. 2013. Droit du travail. Paris: Editions Dalloz. ISBN 978-2-247-11630-0.

LA SEMAINE JURIDIQUE. 2009-4. Actes du colloque de Lyon (2008): La rémunération des fonctionnaires. ISSN 1637-5114.

LARISOVA, M. 2008. Francouzko-cesky/ česko-francouzsky slovnik. Plzen: Ales Cenek. ISBN 978-80-7380-111-3.

LEGISLATION et reglementation en vigueur, la Cour de cassation et du Conseil d'État. Available online: www.legifrance.gouv.fr

MOUNIN, G. 1963. Les problemes theoriques de la traduction. Paris: Gallimard, coll. TEL.

REY, A. 2010. Dictionnaire historique de la langue francaise. Paris: Dictionnaire Robert. ISBN 978-2-84902-997-8.

RIBERT, N. 2013. Dictionnaire pratique social. Paris: Groupe revue fiduciaire. ISBN 978-2-7579-0418-3.

TERRAL, F. 2014. L'empreinte culturelle des termes juridiques. In : Meta : journal des traducteurs / Meta: Translators' Journal, vol. 49, n. 4, pp. 876-890. Available online: http://id.erudit.org/iderudit/009787ar

TRESOR de la Langue française publié par le CNRS. Available online: www.atilf.fr

THIRY, B. 2008. Problemes de jurilinguistique contrastive: les équivalences interlinguistiques en droit. In Cahier de recherche, $N^{\circ}$ 200808/05. CHC ULg Liège. Available online: www.hec.ulg.ac.be/sites/default/files/workingpapers/WP_HECU Lg_20080805_Thiry.pdf

TOMASEK, $\overline{\text { M. }}$ 2003. Preklad v pravni praxi. [Traduction dans la pratique juridique]. Praha: Linde ( $2^{\mathrm{e}}$ édition). ISBN 80-7201-427-7

Zakonik prace, uplne zneni 2015 [Droit du travail tchèque, version complète]. Available online www.pracovnipravo.com/zakonik-prace

http://eurovoc.europa.eu

www.iate.europa.eu

Words: 7740

Characters: 50175 (27, 88 standard pages)

Pierre Brouland

PhDr. Janka Priesolová, CSc.

Department of Romance Languages

Faculty of International Relations

University of Economics

Nám. W.Churchilla 4

13037 Praha 3

Czech Republic

brouland@vse.cz

priesolo@vse.cz 